\title{
Linear Complex Iterative Frequency Estimation of Sparse and Non-Sparse Pulse and Point Processes
}

\author{
Hans-Peter Bernhard and Andreas Springer \\ Johannes Kepler University Linz \\ Institute for Communications Engineering and RF-Systems \\ Altenbergerstr. 69, 4040 Linz, Austria, Email: h.p.bernhard@ ieee.org
}

\begin{abstract}
Clock frequency estimation is a key issue in many signal processing applications, e.g. network clock estimation in wireless sensor networks. In wireless systems or harsh environments, it is likely that clock events can be missed and, therefore, the observed process has to be treated as a sparse periodic process. To parameterize the clock, current research is applying periodogram estimators at a complexity of at least $\mathcal{O}(N \log N)$. We introduce a highly accurate iterative frequency estimator for pulse signals with low computational complexity. An unbiased frequency estimator is presented with a complexity of $\mathcal{O}(N)$. Furthermore the mean square error (MSE), which is proportional to $\mathcal{O}\left(N^{-3}\right)$ is derived and it is shown by theory and simulation that this estimator performs as well as periodogram based methods. The work concludes with simulations on sparse and non-sparse processes including a discussion of the application of the method.
\end{abstract}

\section{INTRODUCTION}

An important task in signal processing is the estimation of the fundamental frequency of pulse signals. Many applications require this task regularly, as there are wireless sensor networks (WSNs), Internet-of-Things (IoT), localization and other similar applications. WSNs need high quality clock estimation e.g. to sample synchronously during offline time, to reduce synchronization discontinuities after long offline time or reducing synchronization error. Usually, high accuracy is linked to high complexity which is a contradiction to low power sensor nodes [1]. Nevertheless, energy efficiency and synchronicity is a vital demand for many systems [2], [3] and, therefore, we present an $\mathcal{O}(N)$ complexity algorithm, albeit state-of-the-art algorithms with the same clock frequency estimation accuracy feature at least a complexity of $\mathcal{O}(N \log N)$ [4]. The algorithm can be used for frequency estimation in sparse and non-sparse processes. Here, we point out the advantages of the algorithm. (i) Low complexity means a fast execution time, also when using simple hardware with low memory requirement. (ii) At every sample interval, one new frequency estimate is available. (iii) Integration of a-priori knowledge to frequency estimation by initializing the iterative algorithm. (iv) The improvement of accuracy or sampling rate of the input signal does not increase computation time, if the number of samples $N$ remains unchanged.

Most other high accuracy methods operate on a frequency domain representation of signals, e.g. Fourier transform, power spectral density estimation and others. In the special case of identifying the fundamental frequency, one of the most common methods is the periodogram $I_{y}(f)$ estimation [5][7] by

$$
\widehat{f}_{P}=\arg \max _{f \in\{\Delta f\}} I_{y}(f) .
$$

Actual research [4] show a variance of the estimation error according to $\mathcal{O}\left(N^{-3}\right)$ at a computational complexity of $\mathcal{O}(N \log (N))$. The spectral range of $\Delta f$ is used to guarantee identifiability [4], [8] and $N$ is the number of samples considered. As we focus on pulse signals, recent research on frequency estimation of cyclostationary signals is relevant. Cyclic spectrum estimation is presented in [9] and [10] that can be used to estimate the fundamental frequency. The MSE is similar to or better than periodogram estimates and is proportional to $\mathcal{O}\left(N^{-2(1+\mu)}\right)$ with $\mu>1 / 3$. As for the periodogram method, there exists a search window $\Delta f$ in the frequency domain which has to be defined previously. In addition to that, a computational complexity of $\mathcal{O}(N \log (N))$ can be reached with this method. In our work, we avoid a maximum search and any frequency transform. Surprisingly, by using only time domain information, we reach the same estimation performance as with frequency domain methods. The work is organized as follows: We give a brief introduction to the signal structure in section II-A and II-B. The estimation method for the sparse process is presented in section III-A, where the estimator itself and its properties are derived, followed by the estimator of the non-sparse process, which is introduced in section III-C as special case of the sparse process estimator. In section IV, the simulations and comparisons with other methods are introduced. Finally, the results are summarized in the conclusion.

\section{PROCESS MODEL}

\section{A. Periodic Pulse Process}

In real world applications, we often observe periodic processes as periodic pulse processes. Therefore, the function space

$$
a(t)=\sum_{n=-\infty}^{\infty} \operatorname{rect}\left(\frac{t-n P+e(t)-\phi}{D}\right)+n(t)
$$

with $t \in \mathbb{R}$ as time variable is considered as basic model. The process parameters are the period $P$ and duration $D$ with $0<D<P<\infty$. $\phi \in \mathbb{R}$ is considered as random phase. $e(t)$ and $n(t)$ are additive phase and amplitude noise 
distributed according to $\mathcal{N}\left(0, \sigma_{e}\right)$ and $\mathcal{N}\left(0, \sigma_{n}\right)$ respectively. We want to estimate the fundamental frequency $f_{P}$ of the process. Therefore, the state changes of the pulse process are used as input of the estimator. The observation of state changes is done by different methods, which are not in the scope of our work. The pulse signal is sampled to gain time discrete measurements. The state changes represent the point process and each state change is recorded with a sampling error and the phase noise $e(t)$. Both sources of error are considered as one compound additive noise to the occurrence of the state change time. A special analysis on sampling or quantization error has been done in [11].

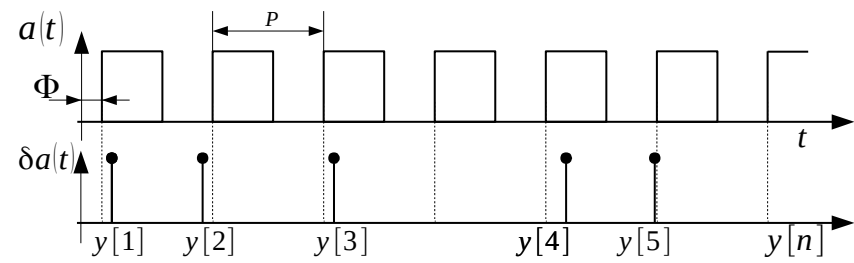

Fig. 1. Pulse and point process to show schematically a noise free pulse process and in parallel a noisy sparse point process.

\section{B. Point Process}

The point process of state changes is represented by a sequence

$$
y[n]=n P+e[n]+\phi
$$

of real numbers with period $P \in] 0, \infty[$ and phase $\phi \in \mathbb{R}$ which has been derived from signals as (2) where the positive edge was used for gathering the consecutive measurements $y[n]$, also see Fig. 1. As with any other observation process, errors may occur and also the problem of missing state changes. Those processes are called "sparse point processes". The special behavior of sparse processes is modeled by randomly recorded periodic events. As random source e.g. a geometric or poisson probability process can be used as illustrated in section III-A.

\section{THE ESTIMATION}

\section{A. The frequency estimator}

This algorithm is based on differences between events to estimate fundamental frequency. Occurring events trigger the sampler, which records $t$, as can be seen in Fig. 2 at the upper sampling switch position. The time $t$ is generated by a local system clock with sufficient high resolution. The sampled signal can be described similarly to a periodic point process, but the simple multiplication of the period $P$ by $n \in \mathbb{N}$ for periodic events (3) is replaced by a multiplication with $x[n]$ as

$$
y[n]=x[n] P+e[n]+\phi
$$

where $e[n]$ is an additive Gaussian measurement noise with $\mathcal{N}\left(0, \sigma_{e}\right)$ and $\phi$ is a random phase. We define $x[n]$ as stochastic random variable with $x[n] \in \mathbb{N}$ and $x[a]>x[b]$ if $a>b$. Hence,

$$
x[n+1]=x[n]+d[n]
$$

where $d[n]$ are i.i.d. with mean $\mu_{d}$ and variance $\sigma_{g}^{2}$. Alterna- tively, $x[n]$ can be written as $x[n]=\sum_{i=1}^{n} d[i]$ and, therefore, the sparse process is given by

$$
y[n]=P \sum_{i=1}^{n} d[i]+e[n]+\phi .
$$

To estimate the fundamental frequency $f_{P}=\frac{1}{P}$ the difference between two events of the point process are used and calculated as depicted in Fig. 2

$$
y_{d}[n]=y[n]-y[n-N]=P \sum_{i=0}^{N-1} d[n-i]+e_{d}[n]
$$

where $e_{d}[n]=e[n]-e[n-N]$. If a trigger event is missing for $t>y[n]+\Delta t_{\max }$ an averaged period is used by $y[n+1]=$ $y[n]+y_{d}[n] /\left\lfloor\mu_{d} N\right\rfloor$ to fill in the gap. $\Delta t_{\max }$ is determined by the smallest frequency of the search window $\Delta f$ as $\Delta t_{\max }=$ $\frac{1}{\Delta f_{\min }}$ and $\mu_{d}$ is the mean of $d[n]$. The difference $y_{d}[n]$ is calculated with $\left\lfloor\mu_{d} N\right\rfloor$ interpolated samples by the estimator in Fig. 2. Thus, we rewrite (7) using $P \sum_{i=0}^{N-1} d[n-i]=$ $P\left\lfloor N \mu_{d}\right\rfloor$ as sum over all interpolated $\left\lfloor\mu_{d} N\right\rfloor$ samples

$y_{d}[n]=P \sum_{i=0}^{N-1}\left(d[n-i]+\frac{e_{d}[n]}{N}\right)=\sum_{i=0}^{\left\lfloor\mu_{d} N\right\rfloor-1}\left(P+\frac{e_{\mu_{d}}[n]}{\left\lfloor\mu_{d} N\right\rfloor}\right)$.

As commonly known, the interpolation does not change the error variance of the original samples. Hence, the interpolated error has to be $e_{\mu_{d}}[n]=\sqrt{\mu_{d}} e_{d}[n]$ to fulfil $\frac{\mu_{d} \sigma_{d}^{2}}{\left|\mu_{d} N\right|}=\frac{\sigma_{d}^{2}}{N}$ per non interpolated sample for all $\mu_{d}$. Consequently, if interpolated samples are considered, $e_{\mu_{d}}[n]$ has to be used. To proceed, we introduce a vector notation as an intermediate step by

$$
\begin{aligned}
\mathbf{y}_{d}^{N}[n] & =\left\{y_{d}[n], \ldots, y_{d}[n-N+1]\right\} \\
& =\left\{P d_{d}^{N}[n]+e_{d}[n], \ldots, P d_{d}^{N}[n-N+1]+e_{d}[n-N+1]\right\},
\end{aligned}
$$

where $d_{d}^{N}[n]=\sum_{i=0}^{N-1} d[n-i] \cdot \mathbf{y}_{d}^{N}[n]$ is the sum of two vectors, the center vector $\mathbf{c}^{N}[n]=\left\{P d_{d}^{N}[n], \ldots, P d_{d}^{N}[n-\right.$ $N+1]\}$ and the error vector of a multivariate Gaussian random variable $\mathbf{e}_{d}^{N}[n]=\left\{e_{d}[n], \ldots, e_{d}[n-N+1]\right\}$. This results in

$$
\mathbf{y}_{d}^{N}[n]=\mathbf{c}^{N}[n]+\mathbf{e}_{d}^{N}[n] .
$$

Hence, $\mathbf{y}_{d}^{N}[n]$ represents a multivariate i.i.d. Gaussian random variable with a diagonal covariance matrix, which is shifted by the center vector $\mathbf{c}^{N}[n]$. All diagonal elements of the covariance matrix have the value $\sigma_{d}^{2}$ and it is therefore invariant to a rotation in the $N$ dimensional space. To emphasize, the variance of in the $N$-dimensional diagonal vector has the same variance as the variance in each of the $N$ dimensions. Hence, the center of the $N$-dimensional observation is given by $\mathbf{c}^{N}[n]$ with an additive error in direction of the vector $\frac{\mathbf{c}^{N}[n]}{\left|\mathbf{c}^{N}[n]\right|}$ with $\mathbf{e}_{d}^{N}[n] \frac{\mathbf{c}^{N}[n]}{\left|\mathbf{c}^{N}[n]\right|}=e_{d}[n]$. We write the vector components as

$$
P d_{d}^{N}[n]=P \sum_{i=0}^{N-1} d[n-i]=P\left\lfloor N \mu_{d}\right\rfloor .
$$

The length of the observed vector of the filled up sparse process can be determined by using the absolute value of $\mathbf{y}_{d}^{N}[n]$ as 


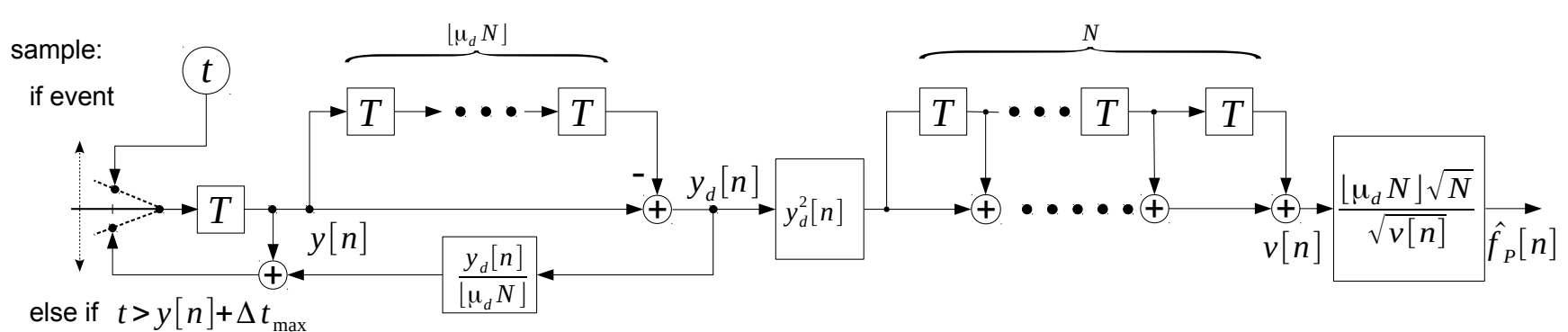

Fig. 2. Iterative Frequency Estimator for $\widehat{f}_{P}(n)$ depicted using an unrolled iteration.

$$
\begin{aligned}
\left|\mathbf{y}_{d}^{N}[n]\right| & =\left|\mathbf{c}^{N}[n]\right|+\mathbf{e}_{\mu_{d}}^{N}[n] \frac{\mathbf{c}^{N}[n]}{\left|\mathbf{c}^{N}[n]\right|} \\
& =\sqrt{\sum_{k=1}^{N}\left(P\left\lfloor N \mu_{d}\right\rfloor\right)^{2}}+e_{\mu_{d}}[n] \\
& =P\left\lfloor\mu_{d} N\right\rfloor \sqrt{N}+e_{\mu_{d}}[n] .
\end{aligned}
$$

Finally, we write for the sparse process

$$
v[n]=\sum_{i=0}^{N-1} y_{d}^{2}[n-i]=\left|\mathbf{y}_{d}^{N}[n]\right|^{2}=\left(P\left\lfloor\mu_{d} N\right\rfloor \sqrt{N}+e_{\mu_{d}}[n]\right)^{2}
$$

and use the estimator structure of Fig. 2, to estimate $f_{P}$ of the sparse process. With (13), the estimator equation is

$$
\hat{f}_{P}^{N}[n]=\frac{\left\lfloor\mu_{d} N\right\rfloor \sqrt{N}}{\sqrt{\sum_{i=0}^{N-1} y_{d}^{2}[n-i]}} .
$$

The implementation can be done extremely efficiently when using

$$
v[n]=\sum_{i=0}^{N-1} y_{d}^{2}[n-i]=v[n-1]-y_{d}^{2}[n-N]+y_{d}^{2}[n]
$$

for consecutive estimates. Therefore, the algorithm is called iterative frequency estimator (IFE).

\section{B. Properties and bounds of the iterative frequency estimator}

Theorem 1. The estimator is unbiased for $N \rightarrow \infty$ with $\left(\hat{f}_{P}^{N}[n]-f_{P}\right) \rightarrow 0$ for a sparse point process.

Proof. $e_{d}[n]$ is a random variable of a stochastic i.i.d. process with finite absolute value $\left|e_{d}[n]\right|<\infty$. Therefore, the supremum of the difference $\left(\hat{f}_{P}^{N}[n]-f_{P}\right)$ is with (14) and (13) given by

$$
\begin{aligned}
\limsup _{N \rightarrow \infty} & \left(f_{P}-\hat{f}_{P}^{N}[n]\right)=\limsup _{N \rightarrow \infty}\left(\frac{1}{P}-\frac{\left\lfloor\mu_{d} N\right\rfloor \sqrt{N}}{P\left\lfloor\mu_{d} N\right\rfloor \sqrt{N}+\sqrt{\mu_{d}} e_{d}[n]}\right) \\
= & \limsup _{N \rightarrow \infty}\left(\frac{1}{P}-\frac{1}{P+\frac{\sqrt{\mu_{d}} e_{\mu_{d}}[n]}{\left\lfloor\mu_{d} N\right\rfloor \sqrt{N}}}\right) \\
= & \limsup _{N \rightarrow \infty}\left(\frac{1}{P}-\frac{1}{P+O\left(N^{-1.5}\right)}\right) \rightarrow 0
\end{aligned}
$$

Theorem 2. The mean square error (MSE) of the frequency estimator for sparse point processes is

$$
E\left[\left(f_{P}-\hat{f}_{P}^{N}[n]\right)^{2}\right] \approx \frac{\sigma_{d}^{2}}{\left\lfloor\mu_{d} N\right\rfloor P^{4} N^{2}}
$$

if $\left|\frac{\sqrt{\mu_{d}} e_{d}[n]}{P\left\lfloor\mu_{d} N\right\rfloor \sqrt{N}}\right| \leq 0.1$ for finite and infinite $N$.

Proof. The MSE of the estimator is given by

$$
E\left[\left(f_{P}-\hat{f}_{P}^{N}[n]\right)^{2}\right]=E\left[\left(\frac{1}{P}-\frac{1}{P} \frac{1}{\left(1+\frac{\sqrt{\mu_{d}} e_{d}[n]}{P\left\lfloor\mu_{d} N\right\rfloor \sqrt{N}}\right)}\right)^{2}\right]
$$

and thus

$$
E\left[\left(f_{P}-\hat{f}_{P}^{N}[n]\right)^{2}\right]=E\left[\frac{1}{P^{2}}\left(\frac{\frac{\sqrt{\mu_{d}} e_{d}[n]}{P\left\lfloor\mu_{d} N\right\rfloor \sqrt{N}}}{\left(1+\frac{\sqrt{\mu_{d}} e_{d}[n]}{P\left\lfloor\mu_{d} N\right\rfloor \sqrt{N}}\right)}\right)^{2}\right] .
$$

The approximation of $f(x)=\frac{x^{2}}{(1+x)^{2}}$ is done by applying the Taylor polynomial $x^{2}-2 x^{3}+R_{4}(x)$ if $x \ll 1$ where $R_{4}$ is the Lagrange residual. When using random variables and considering approximation error bounds, we have to use additional information about the distribution. The distribution of the random variable has to be truncated according to the values of $e_{d}[n]$, which do not exceed the search window of $\pm 0.5 P$. Hence, we can evaluate the Lagrange reminder by using the fourth derivative $\left|f^{(4)}(x)\right|=\left|\frac{72-48 x}{(1+x)^{6}}\right| \leq M=150$ under the weak restriction of $|x|=\left|\frac{\sqrt{\mu_{d}} e_{d}[n]}{P\left\lfloor\mu_{d} N\right\rfloor \sqrt{N}}\right| \leq 0.1$. Finally, we apply the approximation $\tilde{f}(x)=x^{2}-2 x^{3}+150 x^{4}$ to (19)

$$
\begin{array}{r}
E\left[\left(f_{P}-\hat{f}_{P}^{N}[n]\right)^{2}\right] \leq E\left[\frac { 1 } { P ^ { 2 } } \left(\frac{\mu_{d} e_{d}^{2}[n]}{\left\lfloor\mu_{d} N\right\rfloor^{2} P^{2} N}-\frac{\mu_{d} \sqrt{\mu_{d}} 2 e_{d}^{3}[n]}{\left[\mu_{d} N\right]^{3} P^{3} \sqrt{N^{3}}}\right.\right. \\
\left.\left.+150 \frac{\mu_{d}^{2} e_{d}^{4}[n]}{\left[\mu_{d} N\right]^{4} P^{4} N^{2}}\right)\right] .
\end{array}
$$

The third moment $E\left[e_{d}^{3}[n]\right]$ of $e_{d}[n]$ vanishes because the error is zero mean (4) and thus

$$
E\left[\left(f_{P}-\hat{f}_{P}^{N}[n]\right)^{2}\right] \leq E\left[\frac{\mu_{d} e_{d}^{2}[n]}{\left[\mu_{d} N\right]^{2} P^{4} N}\right]+E\left[\frac{150 \mu_{d}^{2} e_{d}^{4}[n]}{\left[\mu_{d} N\right]^{4} P^{6} N^{2}}\right] .
$$


The residual is a strict positive fourth order moment and with $M>0$, we find an upper and lower bound for (21) as

$$
\frac{\mu_{d} \sigma_{d}^{2}}{\left[\mu_{d} N\right\rfloor^{2} P^{4} N} \leq E\left[\left(f_{P}-\hat{f}_{P}^{N}[n]\right)^{2}\right] \leq \frac{\mu_{d} \sigma_{d}^{2}}{\left[\mu_{d} N\right\rfloor^{2} P^{4} N}+\mathcal{O}\left(N^{-6}\right)
$$

where the residual vanishes at least with $\mathcal{O}\left(N^{-6}\right)$ and is therefore

$$
\frac{\sigma_{d}^{2}}{\left\lfloor\mu_{d} N\right\rfloor P^{4} N^{2}} \approx E\left[\left(f_{P}-\hat{f}_{P}^{N}[n]\right)^{2}\right]
$$

The result depends on $e_{d}[n]$ which is the sum of two independent random variables $e[n]$ and $e[n-N]$. Hence we can state that the variances have to be added. As we demonstrate in simulations, the estimation performs according to (22) for a wide range of parameters, but if $\sigma_{e_{d}}^{2}=2 \sigma_{e}^{2}$ exceeds a certain threshold, the estimation quality drops dramatically. As we will see in section IV, the threshold is about $P \approx 5 \sigma_{e_{d}}$. Hence all values of $\sigma_{e}^{2}>\frac{P^{2}}{100}$ will lead to increasing errors based on wrong assignment of events to subsequent periods. We conclude that this threshold represents a limit for pure time domain frequency estimation.

In terms of MSE, our results are similar to that found for the periodogram method [7] up to the threshold discussed above, but with dramatically less complexity. In section IV the method is discussed in more details.

\section{The frequency estimator for non-sparse point processes}

Here we consider the estimator based on sampling of the observed non-sparse point process (3). According to (6), (7) and (8), we derive similar to (12)

$$
\begin{aligned}
\left|\mathbf{y}_{d}^{N}[n]\right| & =\left|\mathbf{c}^{N}[n]\right|+e_{d}[n] \frac{\mathbf{c}^{N}[n]}{\left|\mathbf{c}^{N}[n]\right|} \\
& =\sqrt{\sum_{i=1}^{N} N^{2} P^{2}}+e_{d}[n]=N \sqrt{N} P+e_{d}[n]
\end{aligned}
$$

which gives an equation for the estimator by using (13)

$$
\hat{f}_{P}^{N}[n]=\frac{N \sqrt{N}}{\sqrt{\sum_{i=0}^{N-1} y_{d}^{2}[n-i]}}
$$

similar to (14), but with $\mu_{d}=1$, which is coherent because the process is non-sparse. Between all consecutive samples is one period, obviously the mean value has to be one. Consequently Theorem 1 and Theorem 2 holds with $\mu_{d}=1$.

\section{Computing complexity}

As shown in the previous section we use the same type of estimator for sparse and non-sparse processes. The value of $\mu_{d}$ has to be adapted to the mean of the sparse process. If $\mu_{d}$ is known or if we have a non-sparse process, the estimation algorithm consists of $N$ multiplications, $N$ summations and one square root operation. Therefore, the straightforward complexity is $\mathcal{O}(N)$. If the algorithm is implemented iteratively, the number of operations can be reduced to an initial $\mathcal{O}(N)$ complexity and all consecutive estimates can be done by one

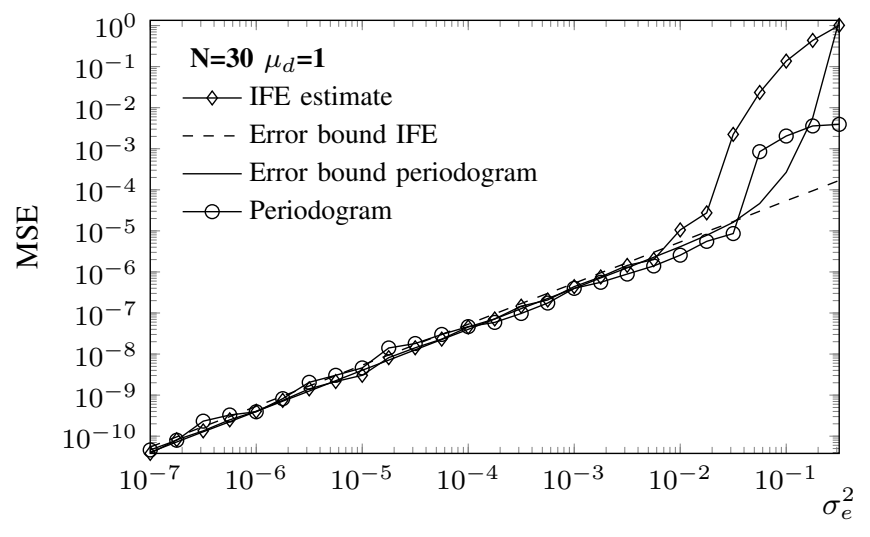

Fig. 3. The MSE of frequency estimation for the time series estimate and as baseline the periodogram estimate of a sparse process with $\mu_{d}=1$.

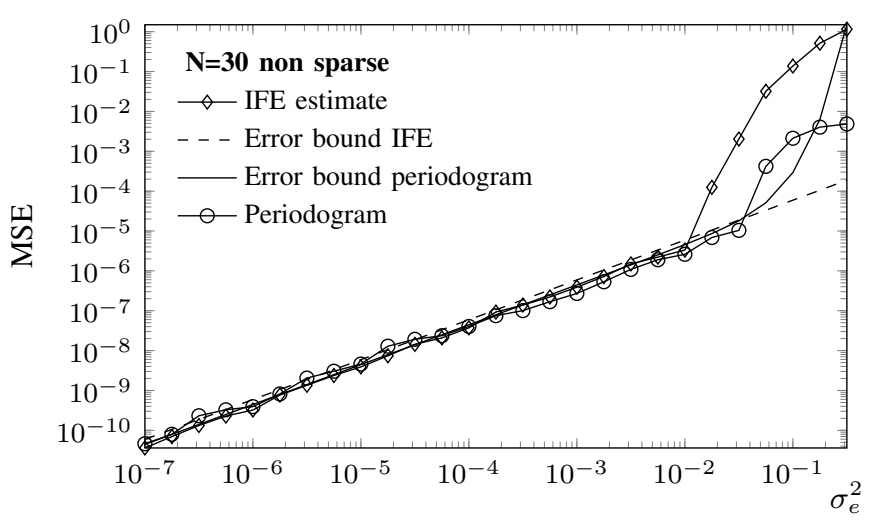

Fig. 4. The MSE of frequency estimation for the time series estimate and as baseline the periodogram estimate of a non-sparse process.

multiplication, two summations and one square root operation $\mathcal{O}(4)$. According to Fig. $2, \mu_{d} N$ is gained by a counter. The counter accumulates the number of samples of both sample switch positions. The counter is stopped when $N$ real events, which means only upper switch position, have occurred.

\section{Simulation RESUlts}

Two algorithms to estimate the frequency of sparse processes are used in the simulations. As baseline algorithm we applied the periodogram, the most efficient and most accurate method so far published [4]. The baseline algorithm was implemented according to [4] with a chirp-z transform of a quantized time series. The frequency estimate was found by the maximum search proposed in [4]. The process data were generated by using a geometrically distributed random variable for the distances $x[n]$ in equation (6). The additive measurement noise $e[n]$ is modeled as Gaussian according to $\mathcal{N}\left(0, \sigma_{e}\right)$. With Monte Carlo simulation, 100 realizations of the process were created as raw data to estimate the frequency for statistical randomized experiments. To allow reliable comparison, we used the same parameter set as it was used in [4]. The period of the generated sparse process is given by $P=\frac{\pi}{3}$ and the initial phase is $\phi=0.2$. Two different mean values of the geometric distribution were used, $\mu_{d}=1$ and $\mu_{d}=10$. Additionally we analyzed the computation 


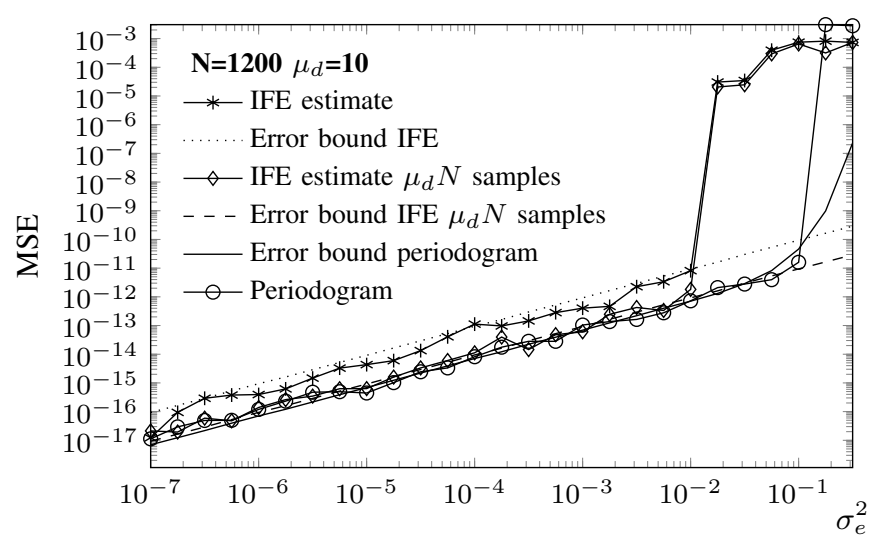

Fig. 5. The MSE of frequency estimation for the time series estimate and as baseline the periodogram estimate of a non-sparse process.

complexity for the baseline algorithm. We considered the most accurate estimation in our work with $N=1200$ and a quantization as recommended by [4], [11] of $q=7.5$. Thus, the complexity is of $\mathcal{O}(q N \log (q N)) \approx 1.2 \cdot 10^{5}$ for one estimate. This number does not include maximum search which can add $\mathcal{O}(10 N)$ on top of it. In contrast to that, our algorithm would take $\mathcal{O}(N)=1.2 \cdot 10^{3}$ operations, which are two orders of magnitude less at comparable performance. In Figures 3 to 6 the MSE is depicted as function of additive Gaussian measurement noise. We consider Fig. 3 and Fig. 4 to compare sparse and non-sparse processes. The MSE is depicted and it can be clearly seen that there is no significant difference and the algorithm performs on both processes in the same way. In both figures the baseline algorithm is also depicted. The baseline algorithm is also used in Fig. 5 to compare both algorithms for a high number of samples $N=1200$ and a sparse process with a mean of $\mu_{d}=10$, which shows similar MSE for both algorithms below the threshold of $5 \sigma_{d}^{2}$. If the IFE uses all $\left\lfloor\mu_{d} N\right\rfloor$, observed and interpolated samples, the MSE of periodogram and IFE algorithm are equivalent even for high $\mu_{d}$. The threshold and its dependency can be seen in Fig. 6 for two different frequencies. It is evident that the threshold is frequency dependent, roughly by $\sigma_{e}^{2}>\frac{P^{2}}{100}$. An detailed study on the threshold has to be done in further research.

\section{CONCLUSION}

We present a fundamental frequency estimator for sparse and non-sparse point processes. The principle relies on a low complex averaging algorithm of squared differences. The simulations show the same MSE as the theoretically derived lower bound which is proportional to $\mathcal{O}\left(N^{-3}\right)$. If we compare our algorithm with a baseline periodogram algorithm, we see that the estimation error is at a similar absolute value but with dramatically less complexity of $\mathcal{O}(N)$ as compared to $\mathcal{O}(N \log (N))$. Furthermore, the algorithm avoids block processing as necessary for the chirp-z transform. This allows us to use it in low power sensor nodes prepared for harsh environments where it is likely to miss clock synchronization pulses. The algorithm can be implemented iteratively to save most of the computing time in subsequent estimates. There are already existing implementations in the area of energy harvest-

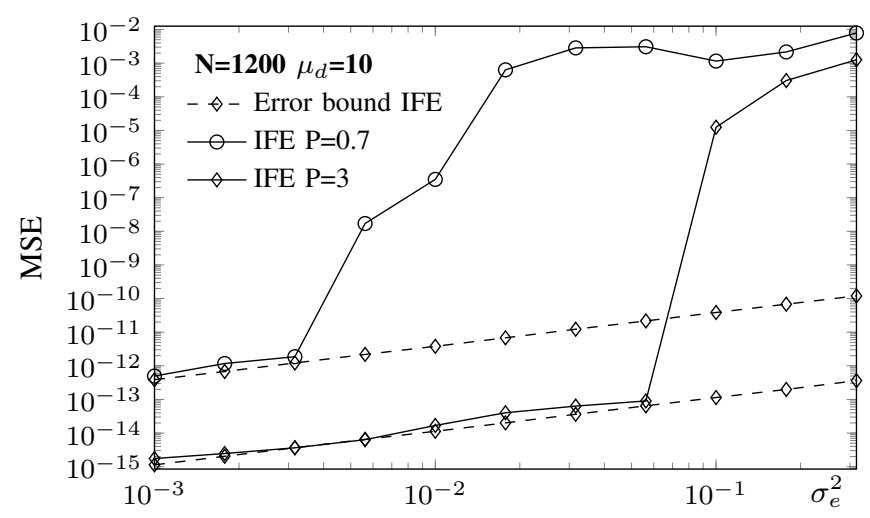

Fig. 6. A detailed view on the estimation of two processes with different fundamental frequencies under bad noise conditions.

ing wireless sensor nodes [2], [3], which benefit from the high estimation accuracy and less computational complexity.

\section{ACKNOWLEDGMENT}

The research from DEWI project (www.dewi-project.eu) leading to these results has received funding from the ARTEMIS Joint Undertaking under grant agreement $n^{o} 621353$ and the Austrian Research Promotion Agency (FFG) under grant no. 842547.

\section{REFERENCES}

[1] H. P. Bernhard, A. Springer, A. Berger, and P. Priller, "Life cycle of wireless sensor nodes in industrial environments," in 2017 IEEE World Conference on Factory Communication Systems (WFCS), 5 2017, Aufsatz / Paper in Tagungsband (referiert), pp. - .

[2] H.-P. Bernhard, A. Berger, and A. Springer, "Analysis of delta-sigmasynchronization in wireless sensor nodes," in Industrial Informatics (INDIN), 2015 IEEE 13th International Conference on, July 2015, pp. 914-918.

[3] H. P. Bernhard, A. Berger, and A. Springer, "Timing synchronization of low power wireless sensor nodes with largely differing clock frequencies and variable synchronization intervals," in 2015 IEEE 20th Conference on Emerging Technologies Factory Automation (ETFA), Sept 2015, pp. $1-7$.

[4] R. G. McKilliam, I. V. L. Clarkson, and B. G. Quinn, "Fast sparse period estimation,” IEEE Signal Processing Letters, vol. 22, no. 1, pp. 62-66, Jan 2015.

[5] E. J. Hannan, "The estimation of frequency," Journal of Applied Probability, vol. 10, no. 3, pp. 510-519, 1973. [Online]. Available: http://www.jstor.org/stable/3212772

[6] B. G. Quinn, "Recent advances in rapid frequency estimation," Digital Signal Processing, vol. 19, no. 6, pp. 942 - 948, 2009, dASP'06 - Defense Applications of Signal Processing. [Online]. Available: http://www.sciencedirect.com/science/article/pii/S1051200408000559

[7] B. G. Quinn, R. G. McKilliam, and I. V. L. Clarkson, "Maximizing the periodogram," in IEEE GLOBECOM 2008 - 2008 IEEE Global Telecommunications Conference, Nov 2008, pp. 1-5.

[8] N. D. Sidiropoulos, A. Swami, and B. M. Sadler, "Quasi-ml period estimation from incomplete timing data," IEEE Transactions on Signal Processing, vol. 53, no. 2, pp. 733-739, Feb 2005.

[9] A. Napolitano, "Asymptotic normality of cyclic autocorrelation estimate with estimated cycle frequency," in Signal Processing Conference (EUSIPCO), 2015 23rd European, Aug 2015, pp. 1481-1485.

[10] _ "On cyclic spectrum estimation with estimated cycle frequency," in Signal Processing Conference (EUSIPCO), 2016 24rd European, Aug 2016, pp. 160-164.

[11] H. Ye, Z. Liu, and W. Jiang, "Efficient maximum-likelihood period estimation from incomplete timing data," in International Conference on Automatic Control and Artificial Intelligence (ACAI 2012), March 2012, pp. 959-962. 